\title{
ENFOQUE PRÁCTICO PARA LA DETERMINACIÓN DE POLÍTICAS DE INVENTARIO CENTRALIZADAS EN UN SISTEMA 1-BODEGA/ N-MINORISTAS A TRAVÉS DE SIMULACIÓN/OPTIMIZACIÓN
}

\author{
$\checkmark$ LiLiana DELGado ${ }^{1}$ \\ HÉCTOR HERNÁN TORO ${ }^{2}$ \\ JUAN JOSÉ BRAVO ${ }^{3}$
}

\section{RESUMEN}

En la administración de la cadena de suministro la colaboración se ha convertido en factor clave de éxito. Estrategias de operación en las cuales cada nodo opera en condiciones óptimas han probado ser inadecuadas, y se ha determinado que es mejor una estrategia de coordinación de la cadena como un todo. Una cadena de 1-Bodega/N-Minoristas típicamente opera con cada minorista haciendo sus pedidos a la bodega de acuerdo con sus propias políticas de inventario. En este artículo se estudia el caso en el que la bodega toma decisiones centralizadas, definiendo las políticas de reabastecimiento del inventario de los minoristas usando la política de revisión periódica $(\mathrm{R}, \mathrm{S}, \mathrm{S})$. La política óptima se determina utilizando una heurística que combina simulación Montecarlo con Optimización, basada en una implementación en hoja de cálculo. El enfoque se prueba en un caso, observándose reducciones en los costos relevantes del inventario cuando se comparan los enfoques centralizado versus el descentralizado.

PALABRAS CLAVES: 1-Bodega/N-Minoristas, Simulación/Optimización, Revisión Periódica, Política de Inventario Conjunta.

\section{A PRACTICAL APPROACH TO DEVELOP CENTRALIZED INVENTORY POLICIES FOR 1-WAREHOUSE/N-RETAILERS SYSTEMS THROUGH SIMULATION/OPTIMIZATION}

\section{ABSTRACT}

Collaboration in supply chain management has become a key success factor. Operational strategies in which each node operates under optimum operating conditions have proven to be inadequate, and it has been determined that a

1 Maestría en Ingeniería con Énfasis en Ingeniería Industrial. Profesora, Escuela de Ingeniería Industrial, Universidad del Valle, Colombia.

2 Ingeniero industrial, Especialista en Logística y M.Sc., en Ingeniería, Universidad del Valle, Colombia.

3 Doctorado en Ingeniería. Magíster en Ingeniería de Sistemas. Universidad del Valle, Colombia.

Autor de correspondencia: Delgado, L. (Liliana): Escuela de Ingeniería Industrial. Calle 13 No. 100 - 00. Edificio 357 - Ciudadela Universitaria Meléndez. Universidad del Valle, Cali, Colombia Tel.: (572) 3212100. Ext. 7006. Correo electrónico: liliana.delgado@correounivalle.edu.co
Historia del artículo:

Artículo recibido: 14-II-2014 / Aprobado: 12-VI-2017

Disponible online: 30 de agosto de 2017

Discusión abierta hasta octubre de 2018 
strategy of coordination of the chain as a whole is best perceived. The One-warehouse N-retailer chain typically operates with each retailer placing orders to the warehouse according to its own inventory policies. In this article we study the case where the warehouse makes centralized decisions, defining retail inventory replenishment policies using the (R, s, S) periodic review policy. The optimal policy is determined using a heuristic that combines Monte Carlo simulation with Optimization, based on an implementation in a spreadsheet scheme. The approach is tested in one case, showing reductions in the relevant inventory costs when centralized versus decentralized approaches are compared.

KEYWORDS: One-warehouse/N- retailers, Simulation/Optimization, Periodic Review, Joint Inventory Policy.

\section{ENFOQUE PRÁTICO PARA A DETERMINAÇÃO DE POLÍTICAS DE INVENTÁRIO CENTRALIZADAS NUM SISTEMA 1-ADEGA/ N-VAREJISTAS ATRAVÉS DE SIMULAÇÃO/OTIMIZAÇÃO}

\section{RESUMO}

$\mathrm{Na}$ administração da corrente de fornecimento a colaboração converteu-se em fator chave de sucesso. Estratégias de operação nas quais a cada nó que opera em condições óptimas de operação têm provado ser inadequadas, e se determinou que é melhor uma estratégia de coordenação da corrente como um tudo. Uma corrente de 1- adega/NVarejistas tipicamente opera com a cada varejista fazendo seus pedidos à adega de acordo a suas próprias políticas de inventário.Neste artigo estuda-se o caso no que a adega toma decisões centralizadas, definindo as políticas de reabastecimento do inventário dos varejistas usando a política de revisão periódica (R,s,S).A política óptima determinase usando uma heurística que combina simulação Montecarlo com Otimização, baseado numa implementação em folha eletrônica.0 enfoque prova-se num caso, observando-se reduções nos Clienteos relevantes do inventário quando se comparam os enfoques centralizado versus o descentralizado.

PALAVRAS-CHAVE: Uma adega N pontos de venda, simulação - otimização, revisão periódica, política de inventário conjunta.

\section{INTRODUCCIÓN}

Uno de los problemas en los que la literatura ha desarrollado un gran interés es el llamado problema 1 Bodega - $\mathrm{N}$ Minoristas (OWNR, por sus siglas en inglés). Este sistema está claramente desarrollado en el artículo escrito por Pukcarnon et al (2014) en el que explican que el sistema OWNR es un sistema de dos etapas que busca determinar las políticas óptimas de reabastecimiento de inventario de los minoristas y la bodega central para que el costo total de inventario correspondiente sea mínimo. Los autores también mencionan que la aleatoriedad de la demanda es un factor cada vez más importante a tener en cuenta.
La metodología más conocida para hacer frente a este tipo de problema es la heurística Schwarz (1973), desde la cual se han desarrollado nuevas heurísticas. Por ejemplo, Abdul-Jalbar et al (2010) y Pukcarnon et al (2014) han propuesto heurísticas que ofrecen resultados con un control más amplio de variables. Algunos artículos se han ocupado de la demanda determinística. Abdul-Jalbar et al. (2010) considera que la demanda del cliente llega a cada tienda minorista a una velocidad constante. Senyigit y Akkan, (2012), presentaron una nueva heurística con la demanda determinística en la que comparan el costo de reposición con el costo de mantener el inventario. También compararon su heurística con 
aquella de Abdul-Jalbar et al (2010), y los resultados computacionales mostraron que la nueva heurística entrega un mejor rendimiento. Por otro lado, Gayon et al (2016) analizó el problema con la demanda determinística donde se les permitió escasez.

El uso de la demanda estocástica fue considerado por Pukcarnon et al., (2014), quienes utilizaron una política de revisión continua de inventario con un algoritmo de optimización de simulación combinada. No es común encontrar artículos en donde se muestre tanto la demanda estocástica y el tiempo de espera, como se va a mostrar en la presente publicación.

En cuanto a la consideración de revisión periódica de las políticas de inventario $(\mathrm{R}, \mathrm{s}, \mathrm{S})$ que se utilizará en las próximas secciones, Monthatipkula y Yenradee (2008) probaron la política de inventario $(\mathrm{R}, \mathrm{s}, \mathrm{S})$ y la contrastaron contra la Planificación de Distribución de Inventario propuesta por ellos. Seleccionaron esta política de revisión periódica debido al buen desempeño que exhibe en términos del costo total del inventario. Esta política de control se describe bien en Whybark y Yang (1996), mientras Schneider et al. (1995) mostraron una buena aproximación para el ajuste de parámetros para esta política de control usando procedimientos de simulación.

El presente artículo se va a enfrentar al problema OWNR tanto con la demanda estocástica y los plazos de entrega bajo una política de inventario de revisión periódica $(\mathrm{R}, \mathrm{s}, \mathrm{S})$, utilizando estrategias de simulación-optimización apoyadas por un algoritmo fácil de implementar. Hasta donde nuestro conocimiento nos permite entender, la estrategia de solución utilizada no ha sido considerada en la literatura todavía para el problema en análisis.

Además, el enfoque general se va a concentrar en la perspectiva centralizada en donde la bodega controla la información sobre la posición de inventario y la demanda de todos los minoristas y productos. Cheng y Zheng (1997) analizaron el problema OWNR con una política de revisión continua centralizada bajo demanda estocástica con un proceso Poisson. Expusieron que muchas empresas, a la luz de la ac- tual tecnología moderna pueden compartir y entregar información de una manera más precisa. Los sistemas bien establecidos de cooperación e información deben estar presentes en este tipo de esquemas. El presente artículo considera que la bodega central tiene suficiente información, así como todas las consideraciones de cooperación necesarias para controlar las políticas de reabastecimiento de inventario de sus minoristas.

La sección 2 presentará el planteamiento del problema, mientras que las secciones 3 , 4 y 5 mostrarán la estrategia heurística utilizada. La sección 6 indicará los resultados que se obtuvieron y finalmente se presentará una conclusión en la sección 7.

\section{PLANTEAMIENTO DEL PROBLEMA}

Este artículo se enfrenta al problema de una bodega que controla las políticas de inventario de los minoristas (clientes), que presenta tanto una demanda estocástica y un tiempo de espera al azar entre la bodega y los minoristas. Ponemos en práctica la Política de Reposición Conjunta o "coordinada" (JRP, Joint Replenishment Policy) con el sistema de revisión de inventario periódico, en el que el periodo de revisión (que se llama R) es la cantidad de tiempo al final del cual la bodega comprueba la posición del inventario de todos los minoristas y productos. Por lo tanto, el sistema es también un problema multiperiodo, multiproducto y la política de control de inventario que hemos seleccionado para aplicar es la $(\mathrm{R}, \mathrm{s}, \mathrm{S})$, donde "s" es el punto de pedido y $S$ es el inventario máximo permitido de un producto en la sede del minorista. La política trabaja con la siguiente dinámica: una vez que el final del periodo de R se ha completado, se revisa entonces la posición del inventario de los productos en cada minorista y se toma una decisión: si el inventario de cualquier producto es inferior al punto de pedido (s) entonces la bodega establece una orden para el producto que es igual a la diferencia entre el inventario máximo (S) y la posición inventario actual en el momento de revisión. 
Figura 1. Procedimiento de simulación Montecarlo

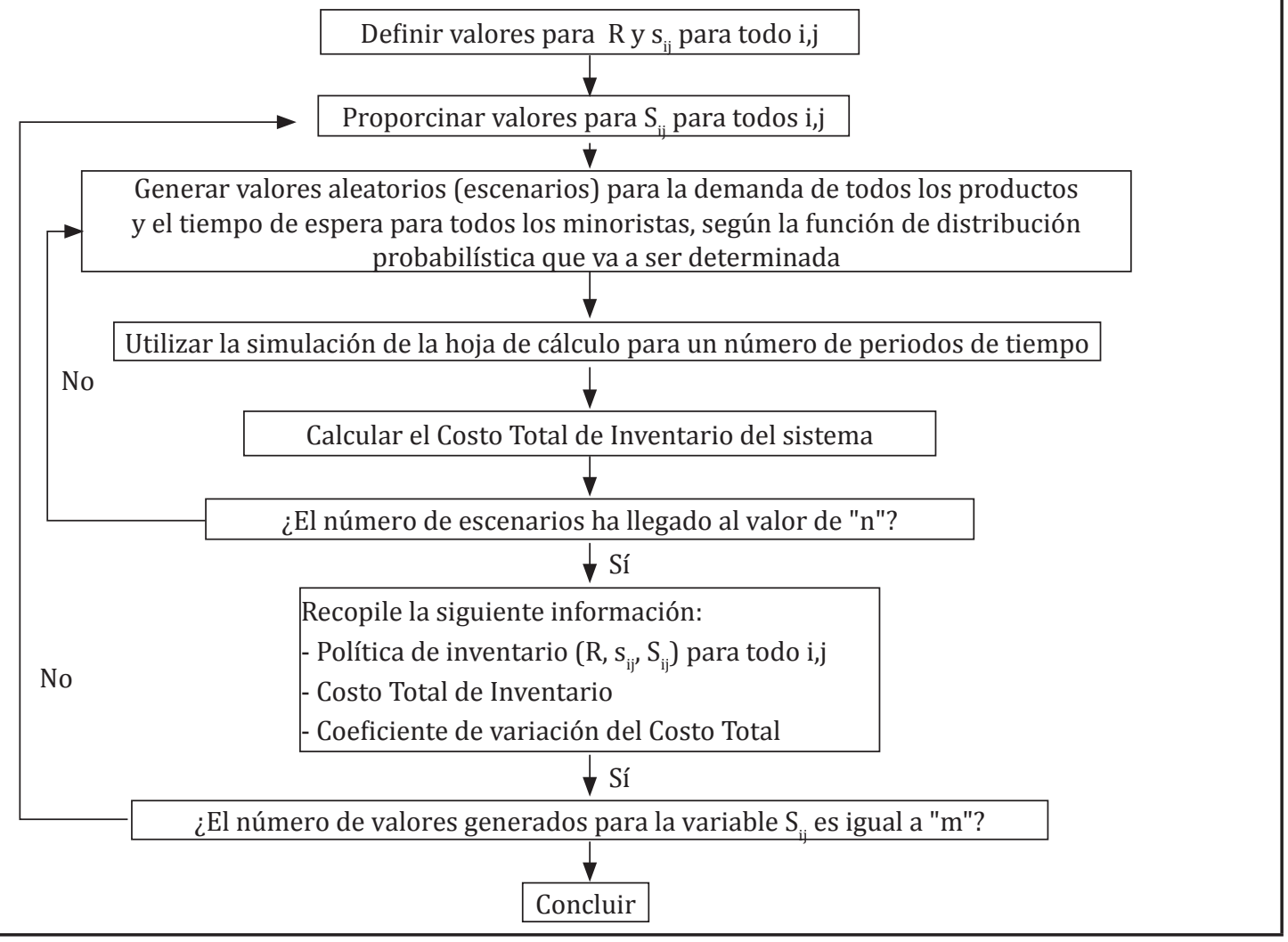

Este sistema puede existir cuando la bodega y los minoristas comparten información sobre dos tipos de conjuntos de datos: la demanda y los niveles de inventario. En este caso, los minoristas se basan en la capacidad de control de la bodega en relación con el sistema de inventario. Por lo tanto, la cuestión central es la estrategia centralizada para gestionar las decisiones de inventario.

Para la bodega, las políticas de inventario óptimas para todos los productos y todos los minoristas pueden conducir a un Costo Total de Inventario mínimo.

Si tomamos "i" como el subíndice referido a los productos y "j" el subíndice de los minoristas, entonces la política óptima debe obtener los valores óptimos de R, sij para todos i, j, y Sij, y también para todo $\mathrm{i}$, j, suponiendo que R es un parámetro común. Sin embargo, determinar los valores óptimos para estos tres tipos de parámetros es una tarea muy compleja
(Silver et al, 1998) y esta dificultad se puede afirmar, en mayor medida, si tenemos en cuenta la naturaleza estocástica del problema en estudio.

En la siguiente sección definimos el procedimiento heurístico utilizado, el cual es una combinación de estrategias de simulación y optimización.

\section{PROCEDIMIENTO GENERAL HEURÍSTICO}

Debido a la complejidad del problema, se propone una heurística que parte de la generación de

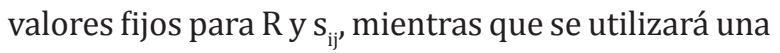
única variable de decisión denominada $\mathrm{S}_{\mathrm{ij}}{ }^{*}$

Con el fin de encontrar el valor óptimo de $S_{i j}$ ' teniendo en cuenta la naturaleza estocástica del problema, se diseñó en primer lugar un procedimiento de simulación Montecarlo con el apoyo de una hoja de cálculo. Básicamente, la hoja de cálculo 
es capaz de calcular, con valores dados para $\mathrm{R}, \mathrm{s}_{\mathrm{ij}} \mathrm{y}$ $\mathrm{S}_{\mathrm{ij}}$, el costo total del inventario para el sistema de inventario de los minoristas, que incluye el costo de mantenimiento del inventario, inventario agotado, $\mathrm{y}$ el costo de pedido.

La Figura 1 ilustra el procedimiento que considera la dinámica Montecarlo donde se generan la demanda y los escenarios de tiempo de entrega.

Según la Figura 1, el procedimiento comienza con los valores de R y s, dados inicialmente. En la siguiente sección se muestra cómo determinar adecuadamente estos valores, que pueden incluso definirse como una decisión administrativa subjetiva. Vale la pena mencionar que el marco de solución es lo suficientemente generalizado para que diferentes valores dados a $\mathrm{R}$ y s puedan ser probados como alternativas.

Observe que "n" es el número de veces que las variables aleatorias tienen que ser generadas para cada conjunto $\mathrm{S}_{\mathrm{ij}}$, mientras que "m" es el número de veces que el conjunto $S_{i j}$ ha de generarse. Debe notarse también que una de las informaciones que se va a recoger es el Coeficiente de Variación del Costo Total, ya que estos datos serán importantes en el proceso de optimización como se verá más adelante.

Figura 2. Procedimiento general de simulación - optimización

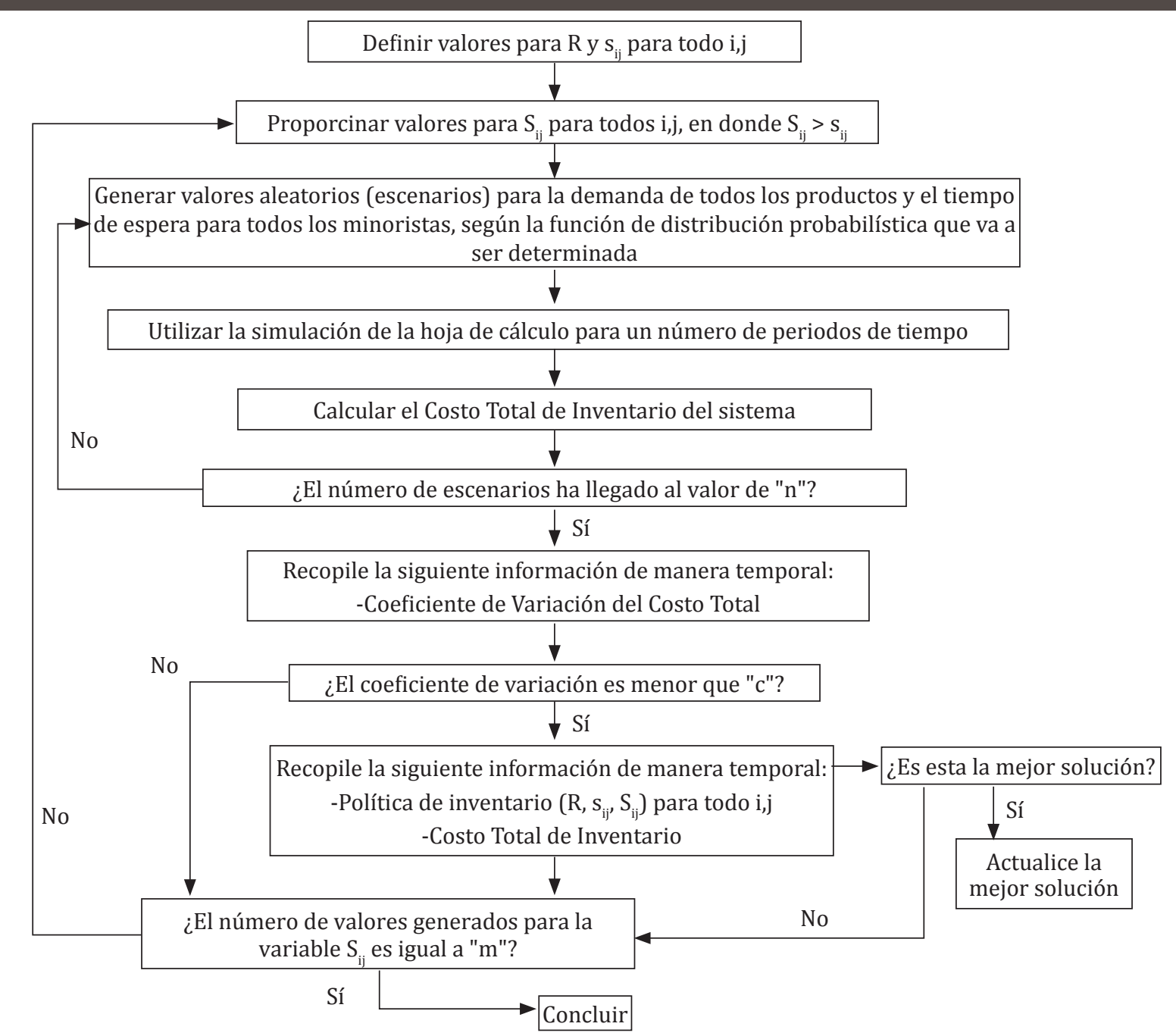


Con la simulación Montecarlo implementada en Excel, la optimización de $\mathrm{S}_{\mathrm{ij}}$ se realizó mediante el uso del software CrystalBall con el optimizador OptQuest que viene con este. El procedimiento de optimización general se representa en la Figura 2.

\section{ACERCA DE LA INICIALIZACIÓN DE $R, s$ y $S$}

El valor de R por lo general se relaciona con los requisitos administrativos, e implica no sólo cuándo revisar el inventario, sino también con qué frecuencia la bodega potencialmente tendría que hacer envíos. Sin embargo, si la compañía no tiene una regla administrativa clara para definirlo, puede ser utilizada la formulación tomada de Silver et al. (1998) y Ballou (2004), como se muestra a continuación:

$$
R=\sqrt{\frac{2 \times \sum_{i}\left(S C_{i}\right)}{\sum_{j} \sum_{i}\left(\mathrm{r} \times \bar{D}_{i j} \times v_{i j}\right)}}
$$

Donde:

SCi: costo de montaje para pedido $i$ [\$/pedido].

r: costo de mantenimiento del inventario [\%/ período de tiempo].

$\bar{D}_{i j}$ : demanda promedio del producto i para cada cliente j durante el horizonte de planificación [unidades/período de tiempo].

vij: costo del producto i para la ubicación del cliente $\mathrm{j}$ [\$/unidad].

Obsérvese que la Ecuación (1) incluye todos los productos utilizados por todos los clientes gracias al control que la bodega central tiene para todo el inventario. Esto sólo es posible en el caso de un esquema centralizado. Sin embargo, podría tener otras estrategias de revisión posibles a ser implementadas en virtud de decisiones centralizadas de inventario.

Por otro lado, el valor del punto de pedido s puede ser visto como un proxy del inventario de seguridad, que es a su vez una función del nivel de servicio requerido y la variabilidad que el sistema tiene que enfrentar. Una vez más, es posible definir s teniendo en cuenta los requisitos administrativos (nivel de servicio).

Por otra parte, con el fin de definir analíticamente el valor inicial de $\mathrm{S}$, basado en los supuestos tanto de la demanda estocástica como de los plazos de entrega, se pueden utilizar las siguientes Ecuaciones (2), (3) y (4), la última propuesta por Ross (1993) para los procesos estocásticos compuestos:

$$
\begin{gathered}
S=E(w)+k^{*} \hat{\sigma}_{w} \\
E(w)=[R+E(L T)] \times E(D) \\
\hat{\sigma}_{w}=\sqrt{R \times E(L T)} \times \hat{\sigma}_{1}^{2}+[E(D)]^{2} \times \hat{\sigma}_{L T}^{2}
\end{gathered}
$$

Donde:

$\mathrm{E}(\mathrm{w})$ : valor esperado de la demanda entre dos momentos de revisión consecutivos (unidades)

$\mathrm{k}$ : factor de seguridad para el nivel de servicio deseado (véase Silver, 1998)

$\hat{\sigma}_{w}$ : desviación estándar de la demanda entre dos momentos de revisión consecutivos (unidades)

R: período de revisión de inventario (unidades de tiempo)

E(LT): valor esperado del tiempo de espera (unidades de tiempo)

E(D): valor esperado de la tasa de demanda (unidades por unidad de tiempo)

$\hat{\sigma}_{1}$ : desviación estándar de los errores de predicción de la demanda (unidades)

$\hat{\sigma}_{L T}$ : desviación estándar del tiempo de espera (unidades de tiempo)

\section{ANTECEDENTES ANALÍTICOS}

En esta sección se muestran las ecuaciones que soportan la simulación-optimización de la hoja de cálculo. En primer lugar, se definen los parámetros estocásticos:

$\mathrm{D}_{\mathrm{ijt}}$ : la demanda del producto $i$ para el cliente $j$ en el período $t$ [unidades].

$\mathrm{LT}_{\mathrm{ijt}}$ : tiempo de espera del producto $i$ para el cliente $j$ para pedidos generados en el período $t$ [días]. 
El Costo de Inventario ( $\mathrm{IC}_{\mathrm{ij}}$ ) se compone de varios componentes, como se muestra a continuación:

$$
\begin{gathered}
I C_{i j t}=O C_{i j t}+H C_{i j t}+S t C_{i j t} \\
O C_{i j t}=S C_{i} \times B_{t} \quad \forall i j t
\end{gathered}
$$

En donde,

$O C_{i j t}=$ costo de pedido para el producto $i \mathrm{y}$ el cliente $j$ en el período $t$ [\$]

$H C_{i j t}=$ costo de mantener el inventario para el producto $i$ y el cliente $j$ en el período $t[\$]$

$S t C_{i j t}=$ costo por falta de existencias para el producto $i$ y el cliente $j$ en el período $t$ [\$]

$S C_{i}=$ costo de montaje para el pedido del producto $i[\$ /$ pedido]

$B_{t}=$ una variable binaria que toma el valor 1 cuando los productos son ordenados en el período $t$, y 0 en caso contrario.

La decisión sobre el pedido de cada producto depende de la política de control de inventario. Para el caso de un sistema $(R, S, S)$ si la posición del inventario se comprueba en un periodo $t$, y si está en o por debajo del punto de s pedido, se realiza el pedido. El tamaño del pedido corresponde a la diferencia entre S y la posición de inventario. La posición de inventario se calculó con la siguiente expresión:

$$
I P_{i j t}=I O H_{i j t}+O O_{i j t}-B O_{i j t} \quad \forall i j t
$$

En donde,
$I P_{i j t}=$ posición de inventario para el producto $i$ para el cliente $j$ y el periodo $t$ [unidades]

$I O H_{i j t}=$ inventario disponible del producto $i$ en al cliente $j$ y el periodo $t$ [unidades]

$O O_{i j t}=$ inventario en pedido del producto $i$ pendiente a llegar al cliente $j$ en el período $t$ [unidades]

$B O_{i j t}=$ pedido pendiente o en espera de producto $i$ en el minorista $j$ en el período $t$ [unidades]

$$
I O H_{i j t}=I O H_{i j t-1}+S R_{i j t}-B O_{i j t-1}-D_{i j t} \quad \forall i j t
$$

En donde,

$S R_{i j t}=$ Recibos programados del producto $i$ que llegan al cliente $j$ al principio del período $t$ [unidades]

$$
S R_{i j t}=\sum_{z=1}^{z=t-1} P O_{i j z} \forall i j z: z+L T(j)=t y t>1
$$

En donde:

$P O_{i j z}=$ orden de producto $i$ registrada para el cliente $j$ en el período $z$ [unidades]

La Figura 3 se utiliza para ilustrar el significado de $P O_{i j z}$ y $S R_{i j t}$ que acabamos de presentar. La línea numerada como 1 en la Figura 3 muestra un orden planificado que se realizó en el período 1 a un cliente determinado. En este momento la orden se llama PO, pero cuatro días más tarde la orden llega a la ubicación del cliente para convertirse en un SR. Otros alternativas posibles se ilustran también para explicar mejor el cálculo de las órdenes que llegan a cierto período de tiempo.

Figura 3. Ejemplo de un recibo programado de producto $i$ llegando al cliente $j$ al principio del período $t=5$.

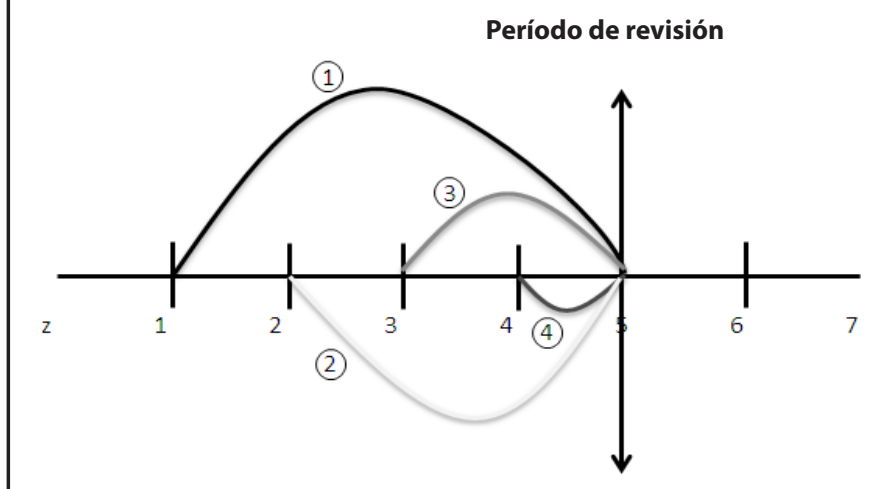

(1) La orden se registró en el periodo 1 con un $\mathrm{LT}=4$ días (2) La orden se registró en el periodo 2 con un $\mathrm{LT}=3$ días (3) La orden se registró en el periodo 3 con un $\mathrm{LT}=2$ días (4) La orden se registró en el periodo 4 con un LT= 1 día 
Siguiendo la política de inventarios, si una orden tiene que ser hecha, el tamaño del pedido es:

$$
P O_{i j t}=S_{i j}-I P_{i j t} \quad \forall i j t
$$

Las órdenes pendientes en $t$, se definen como:

$$
O O_{i j t}=\sum_{z=1}^{z=t-1} P O_{i j z} \forall i j z: z+L T(j)>t y t>1
$$

Nótese que en la Ecuación (9) teníamos z:z+LT(j)=t. En cambio, en la Ecuación (11) tenemos z:z+LT(j)>t, porque $O O_{i j t}$ significa que la orden no ha llegado aún al período de tiempo t.

El costo de mantener inventario se expresa como:

$$
\begin{array}{cc}
H C_{i j t}=h c_{i j} \times I O H_{i j t} & \forall i j t \\
h c_{i j}=v_{i j} \times r & \forall i j
\end{array}
$$

En donde,

$h c_{i j}=$ costo de almacenamiento en el sitio del cliente $j$ para cada unidad de producto $i$ [\$/unidad].

$v_{i j}=$ costo del producto $i$ para el cliente $j[\$ /$ unidad].

$r=$ tasa de costo de inventario [\%/día].

Finalmente, el costo de desabastecimiento se define por la siguiente expresión:

$$
S t C_{i j t}=s t c_{i} \times B O_{i j t} \quad \forall i j t
$$

En donde,

$s t c_{i}=$ costo para cada unidad que no ha sido entregado aún [\$/unidad].

En cuanto al procedimiento de optimización, la única variable que se definió para ser optimizada fue:

$S_{i j}=$ nivel máximo de inventario de producto $i$ para el cliente $j$ [unidades].

\section{Función objetivo}

$$
\text { Minimizar } \quad E(T C)=\sum_{t} \sum_{j} \sum_{t} I C_{i j t}
$$

Sujeto a :

$$
S_{i j} \geq S_{i j} \quad \forall i j
$$

$$
\begin{gathered}
C V_{-} T C \leq C \\
S_{i j} \in Y \quad \forall i j
\end{gathered}
$$

En donde,

$E(T C)=$ costo total esperado $[\$]$

$I C_{i j t}=$ costo de inventario para producto $i \mathrm{y}$ el cliente $j$ en el período $t[\$]$

$S_{i j}=$ punto de pedido para el producto $i$ para el cliente $j$ [unidades]

$C V_{-} T C$ = coeficiente de variación del costo total $c=$ máximo coeficiente de variación permitido

$\mathrm{Y}=$ representa una región definida por otras restricciones del sistema de inventario bajo estudio

Expresiones (16) y (17) se consideraron en la Figura 2. La restricción (17) impone un valor máximo para el coeficiente de variación del costo total, garantizando que el valor mínimo medio de la función objetivo se encuentra también a un valor estable. Recordemos que algunos parámetros son aleatorios (demanda y plazos de entrega) y por lo tanto la función objetivo es una variable aleatoria, y se optimiza su valor medio definiendo varios escenarios para los parámetros aleatorios. Para una política de inventario específica, si generamos demandas y los tiempos de entrega siguiendo sus distribuciones de probabilidad correspondientes es posible generar una distribución de probabilidad empírica para el costo total.

\section{EXPERIMENTOS COMPUTACIONALES}

Se implementó el modelo para un estudio de caso con 15 clientes, una bodega central, 9 tipos de productos y un horizonte de planificación de 7 días. El valor de R obtenido usando la Ecuación (15) fue de 1 día. Sin pérdida de generalidad, se seleccionaron algunas distribuciones de probabilidad. La distribución normal se utilizó para simular valores de demanda, mientras que los tiempos de entrega se modelaron por distribuciones discretas empíricas. El modelo de simulación tiene 1.830 variables estocásticas de ingreso y una variable de salida, así como 
135 variables de decisiones (resultantes de organizar 9 productos para cada uno de los 15 clientes, es decir 9x15=135 variables), 136 limitaciones (el límite superior de cada variable de decisión más el límite superior en el coeficiente de variación de la función objetivo) y 1 función objetivo (el costo total).

Se consideraron seis escenarios, como se muestra en la Tabla 1.

En la Tabla 1, el primer escenario es la base uno, y el resto se utiliza para hacer un contraste y proporcionar algunas conclusiones relevantes. Se observa que otra política de inventario debe probarse: la tradicional $(\mathrm{R}, \mathrm{S})$ en donde el inventario se revisa cada $\mathrm{R}$ unidades de tiempo, una orden siempre se registra y su tamaño corresponde a la diferencia entre el inventario máximo (S) y la posición actual de inventario. Los escenarios (d), (e) y (f) se consideran como un proxy de un enfoque descentralizado, donde cada cliente define su propio momento para revisar la posición de inventario.

Un conjunto de datos hipotéticos se utilizó para probar el procedimiento y la moneda se estableció en pesos colombianos. La tasa de interés se fijó en el 24\% anual. Los plazos de entrega se generaron utilizando una distribución uniforme discreta con límites inferior y superior iguales a 2 y 5 días, respectivamente. El costo de pedido es igual a \$31.285. La Tabla 2 proporciona información de costo asociado a los productos analizados. La Tabla 3 muestra la demanda diaria media y el coeficiente de variación (en paréntesis) para cada par cliente/producto.

Utilizando Crystal Ball y su complemento OptQuest, el procedimiento de optimización se realizó para 1.000 iteraciones, cada una de ellas con un tamaño de muestra de 100 escenarios de los parámetros estocásticos. Los resultados se presentan en las Tablas 4 y 5.

TABLA 1. DEFINICIÓN DE ESCENARIOS

\begin{tabular}{|c|c|c|c|c|c|}
\hline Esc. & $\begin{array}{l}\text { Política de } \\
\text { inventario }\end{array}$ & Revisión del enfoque & Valor inicial de $S_{i j}$ & $\begin{array}{c}\text { Límite } \\
\text { inferior } \\
{\text { para } S_{i j}}\end{array}$ & $\begin{array}{c}\text { Límite superior } \\
\text { para } S_{i j}\end{array}$ \\
\hline $\begin{array}{l}\text { (a) } \\
\text { Base }\end{array}$ & $(\mathrm{R}, \mathrm{S}, \mathrm{S})$ & \multirow{3}{*}{$\begin{array}{l}\text { Todos los clientes, } \\
\text { todos los productos al } \\
\text { mismo tiempo }\end{array}$} & \multirow{2}{*}{$\begin{array}{l}\mathrm{S}_{\mathrm{ij}}=\mathrm{s}_{\mathrm{ij}}+\mathrm{EOQ}_{\mathrm{ij},} \text { para todos } \\
(\mathrm{i}, \mathrm{j}) \\
E O Q_{i j} \\
=\sqrt{\frac{2 \times S C_{i} \times \bar{D}_{i j}}{v \times r}}\end{array}$} & \multirow[b]{2}{*}{$\mathrm{S}_{\mathrm{ij}}$} & \multirow{6}{*}{$\begin{array}{l}\text { disponibilidad de } \\
\text { almacenamiento }\end{array}$} \\
\hline (b) & $(\mathrm{R}, \mathrm{S})$ & & & & \\
\hline (c) & $(\mathrm{R}, \mathrm{S})$ & & Ecuación (2) & $\begin{array}{c}\text { Ecuación } \\
\text { (3) }\end{array}$ & \\
\hline (d) & $(\mathrm{R}, \mathrm{s}, \mathrm{S})$ & \multirow{3}{*}{$\begin{array}{l}\text { R se calcula para cada } \\
\text { cliente de manera } \\
\text { independiente }\end{array}$} & \multirow{2}{*}{$\begin{array}{l}\text { Sij = sij + EOQij, para todos } \\
(\mathrm{i}, \mathrm{j}) \\
E O Q_{i j} \\
\quad=\sqrt{\frac{2 \times S C_{i} \times \bar{D}_{i j}}{v \times r}}\end{array}$} & \multirow[b]{2}{*}{$\mathrm{S}_{\mathrm{ij}}$} & \\
\hline (e) & $(\mathrm{R}, \mathrm{S})$ & & & & \\
\hline (f) & $(\mathrm{R}, \mathrm{S})$ & & Ecuación (2) & $\begin{array}{c}\text { Ecuación } \\
\text { (3) }\end{array}$ & \\
\hline
\end{tabular}




\begin{tabular}{|c|c|c|cc|}
\hline TABLA 2.INFORMACIÓN SOBREEL COSTO DELPRODUCTO \\
Producto & $\begin{array}{c}\text { Costo } \\
\text { unitario }\end{array}$ & $\begin{array}{c}\text { Margen de } \\
\text { utilidad }\end{array}$ & $\begin{array}{c}\text { Costo de } \\
\text { desabastecimiento }\end{array}$ \\
\hline P01 & $\$ 16.780$ & $50 \%$ & $\$ 3.366$ \\
P02 & $\$ 110.279$ & $32 \%$ & $\$ 10.444$ \\
P03 & $\$ 116.447$ & $60 \%$ & $\$ 4.944$ \\
P04 & $\$ 145.540$ & $32 \%$ & $\$ 13.784$ \\
P05 & $\$ 10.194$ & $55 \%$ & $\$ 2.498$ \\
P06 & $\$ 102.157$ & $40 \%$ & $\$ 13.681$ \\
P07 & $\$ 26.455$ & $28 \%$ & $\$ 2.073$ \\
P08 & $\$ 97.199$ & $57 \%$ & $\$ 25.826$ \\
P09 & $\$ 25.234$ & $49 \%$ & $\$ 4.864$ \\
\hline
\end{tabular}

La Tabla 4 presenta el costo total para cada escenario, antes y después de ejecutar el procedimiento de optimización. Se observa que se obtuvo el costo total mínimo para el escenario base como se esperaba. La Tabla 3 también ilustra los ahorros que se pueden lograr mediante la ejecución del proceso de optimización.

La Tabla 5 muestra los resultados cuando se cambia de un enfoque descentralizado a uno centralizado. Para el escenario en el que se permite a cada cliente definir sus políticas de inventario el valor de $R$ varía de 3 a 8 días.

\section{TABLA 3. INFORMACIÓN DE LA DEMANDA}

Productos

\begin{tabular}{c|c|c|c|c|c|c|c|c|c} 
& \multicolumn{10}{|c}{ Productos } \\
\hline Cliente & P01 & P02 & P03 & P04 & P05 & P06 & P07 & P08 & P09 \\
\hline Cliente01 & $5(0,2)$ & $1(0,4)$ & $2(0,5)$ & $1(0)$ & $1(0,2)$ & $1(0)$ & $1(0,2)$ & $1(0)$ & $1(0,2)$ \\
\hline Cliente02 & $60(0,45)$ & $4(0,5)$ & $23(0,13)$ & $1(0,3)$ & $10(0,2)$ & $1(0,2)$ & $4(0,5)$ & $1(0)$ & $4(0,25)$ \\
\hline Cliente03 & $54(0,46)$ & $3(0,67)$ & $21(0,33)$ & $1(0,2)$ & $9(0,11)$ & $1(0,2)$ & $3(0,33)$ & $1(0,2)$ & $4(0,5)$ \\
\hline Cliente04 & $200(0,17)$ & $11(0,09)$ & $78(0,42)$ & $2(0,5)$ & $33(0,52)$ & $1(0,3)$ & $11(0,36)$ & $1(0,3)$ & $14(0,14)$ \\
\hline Cliente05 & $84(0,02)$ & $5(0,2)$ & $33(0,45)$ & $1(0,4)$ & $14(0,29)$ & $1(0,3)$ & $5(0,2)$ & $1(0,2)$ & $6(0,17)$ \\
\hline Cliente06 & $242(0,49)$ & $14(0,07)$ & $94(0,13)$ & $3(0,67)$ & $40(0,1)$ & $1(0,4)$ & $13(0,23)$ & $1(0,3)$ & $17(0,47)$ \\
\hline Cliente07 & $280(0,3)$ & $16(0,19)$ & $109(0,15)$ & $3(0,67)$ & $46(0,5)$ & $1(0,4)$ & $15(0,27)$ & $1(0)$ & $19(0,26)$ \\
\hline Cliente08 & $38(0,45)$ & $3(0,33)$ & $15(0,33)$ & $1(0,2)$ & $7(0,14)$ & $1(0,4)$ & $3(0,67)$ & $1(0,4)$ & $3(0,33)$ \\
\hline Cliente09 & $31(0,35)$ & $2(0,5)$ & $12(0,42)$ & $1(0,3)$ & $6(0,33)$ & $1(0,4)$ & $2(0,5)$ & $1(0,2)$ & $3(0,33)$ \\
\hline Cliente10 & $342(0,41)$ & $19(0,47)$ & $133(0,21)$ & $4(0,25)$ & $57(0,46)$ & $2(0)$ & $19(0,53)$ & $2(0,5)$ & $23(0,04)$ \\
\hline Cliente11 & $256(0,34)$ & $5(0)$ & $99(0,48)$ & $3(0,67)$ & $43(0,12)$ & $1(0,2)$ & $14(0,21)$ & $1(0)$ & $7(0)$ \\
\hline Cliente12 & $394(0,37)$ & $22(0,45)$ & $153(0,08)$ & $4(0,25)$ & $65(0,26)$ & $2(0,5)$ & $21(0,38)$ & $2(0)$ & $27(0,22)$ \\
\hline Cliente13 & $319(0,14)$ & $18(0,39)$ & $124(0,08)$ & $4(0,25)$ & $53(0,25)$ & $2(0)$ & $17(0,47)$ & $1(0,4)$ & $22(0,36)$ \\
\hline Cliente14 & $64(0,31)$ & $4(0,5)$ & $25(0,28)$ & $1(0,3)$ & $11(0,55)$ & $1(0,4)$ & $4(0,5)$ & $1(0,4)$ & $5(0,6)$ \\
\hline Cliente15 & $145(0,42)$ & $8(0,25)$ & $56(0,07)$ & $2(0,5)$ & $24(0,5)$ & $1(0,3)$ & $8(0,5)$ & $1(0,3)$ & $10(0,5)$ \\
\hline
\end{tabular}

TABLA 4. COSTO TOTAL RESULTANTE DEL ENFOQUE INTEGRADO DE SIMULACIÓN-OPTIMIZACIÓN

\begin{tabular}{|l|r|r|r|r|r|r|} 
Escenarios & (a) & (b) & (c) & (d) & (e) & \multicolumn{1}{c|}{ (f) } \\
\hline $\begin{array}{l}\text { Resultados con } \\
\text { Simulación } \\
\text { Montecarlo solamente }\end{array}$ & $\mathbf{1 0 3 . 8 6 6 . 3 2 0}$ & 116.076 .898 & 177.615 .022 & 183.400 .481 & 225.750 .591 & 460.211 .495 \\
\hline $\begin{array}{l}\text { Simulación - enfoque } \\
\text { de optimización }\end{array}$ & $\mathbf{8 2 . 6 0 1 . 2 6 4}$ & 98.310 .391 & 115.320 .378 & 122.780 .341 & 98.328 .361 & 115.410 .191 \\
\hline Ahorros & $\mathbf{2 0 , 4 7 \%}$ & $\mathbf{1 5 , 3 1 \%}$ & $\mathbf{3 5 , 0 7 \%}$ & $\mathbf{3 3 , 0 5 \%}$ & $\mathbf{5 6 , 4 4 \%}$ & $\mathbf{7 4 , 9 2 \%}$ \\
\hline
\end{tabular}


TABLA 5. AHORROS RESULTANTES CUANDO SE CAMBIA DE ESCENARIOS DESCENTRALIZADOS A CENTRALIZADOS

\begin{tabular}{|l|c|c|c|}
\hline \multicolumn{1}{|c|}{ Cambio de escenarios } & De (d) a (a) & De (e) a (b) & De (f) a (c) \\
\hline Ahorros con Simulación Montecarlo solamente & $43,37 \%$ & $48,58 \%$ & $61,41 \%$ \\
\hline Ahorro utilizando Simulación - enfoque de optimización & $32,72 \%$ & $0,02 \%$ & $0,08 \%$ \\
\hline
\end{tabular}

Las Tablas 4 y $\mathbf{5}$ muestran que bajo una estrategia de revisión periódica de inventarios, el enfoque centralizado muestra un mejor rendimiento, y que la política $(\mathrm{R}, \mathrm{S}, \mathrm{S})$ es la más relevante en términos de costos con el mismo nivel de servicio. Por otra parte, el uso de estrategias de simulación-optimización como una metodología combinada conduce a mejores soluciones con grandes ahorros en comparación con las estrategias de simulación Montecarlo.

\section{CONCLUSIONES}

Este artículo analiza la definición de políticas de reabastecimiento de inventario bajo el problema de 1-Bodega/N-Minoristas, que trabaja con la política de revisión periódica $(\mathrm{R}, \mathrm{s}, \mathrm{S})$ bajo un enfoque centralizado, en donde la bodega controla las estrategias de política de inventario de los minoristas. Los resultados muestran que el enfoque centralizado exhibe un mejor rendimiento que uno descentralizado, y también que la estrategia de revisión periódica $(\mathrm{R}, \mathrm{s}, \mathrm{S})$ se comporta mejor que la política $(\mathrm{R}, \mathrm{S})$ implementada tradicionalmente. En cuanto al método de simulación-optimización utilizado en este documento, los resultados han demostrado que las soluciones pueden implicar importantes ahorros que van desde $20 \%$ a $75 \%$ frente al caso en donde sólo se utiliza la simulación Montecarlo. Investigaciones futuras pueden hacerse mediante la exploración de nuevos casos, con el fin de aclarar los beneficios que las estrategias de simulación-optimización pueden obtener. Además, este marco de investigación puede servir para otros tipos de investigación, tal vez concentrados en diferentes tipos de políticas de inventario, tales como políticas de revisión continua.

\section{REFERENCIAS}

Abdul-Jalbar, B., Segerstedt, A., Sicilia, J., \& Nilsson, A. (2010). A new heuristic to solve the one-warehouse N-retailer problem. Computers \& Operations Research, 37(2), 265-272.

Ballou, R.H. (2004). Logistica: administración de la cadena de suministro, Pearson Education.

Chen, F, and Zheng, Y, (1997). One-warehouse multi-retailer systems with centralized stock information. Operations Research, 45 (2), 275-287.

Gayon, J., Massonnet, G., Rapine, C., \& Stauffer, G. (2016). Constant approximation algorithms for the one warehouse multiple retailers problem with backlog or lost-sales. European Journal of Operational Research, 250(1), 155-163

Monthatipkula, C., and Yenradee, P (2008). Inventory/distribution control system in a one-warehouse/multi-retailer supply chain. Int. J. Production Economics 114 (2008) 119-133.

Pukcarnon, V., Chaovalitwongse, P., \& Phumchusri, N. (2014). The Can-Order Policy for One-Warehouse N-Retailer Inventory System: A Heuristic Approach. Engineering Journal, 18(4), 53-72.

Schneider, H., Rinks, D.B., Kelle, P., 1995. Power approximations for a two-echelon inventory system using service levels. Production and Operations Management 4, 381-400.

Schwarzt, L. B. (1973). A simple continuous review deterministic One Warehouse N retailer Inventory Problem. Management Science, 19, 555-566.

Senyigit, E., \& Akkan, H. (2012). A New Heuristic for one Warehouse and N Retailers Problem. Procedia - Social and Behavioral Sciences, 62, 656-660.

Silver, E. A., D. F. Pyke, et al. (1998). Inventory management and production planning and scheduling, Wiley New York,

Whybark, D.C., Yang, S., 1996. Positioning inventory in distribution systems. International Journal of Production Economics 45, 271-278.

\section{PARA CITAR ESTE ARTÍCULO TO REFERENCE THIS ARTICLE / PARA CITAR ESTE ARTIGO /}

Delgado, L.; Toro, H.H.; Bravo, J.J. (2017). Enfoque práctico para la determinación de políticas de inventario centralizadas en un sistema 1-Bodega/N-Minoristas a través de simulación/optimización. Revista EIA, 14(27), enero-junio, pp. 31-41. [Online]. Disponible en: https://doi. org/10.24050/reia.v14i27.1159 